\title{
REVIEW
}

\section{Grazing incidence small-angle neutron scattering: challenges and possibilities}

\author{
Peter Müller-Buschbaum
}

The enhancement of surface sensitivity by grazing incidence geometry facilitates the investigation of nanostructures in thin films and at surfaces. The technique provides information about the surface roughness, lateral correlations, sizes and shapes of objects (such as, nanoparticles and nanostructures) positioned on top of the surface or in a region near the surface. Grazing incidence small-angle neutron scattering (GISANS) overcomes the limitations of conventional small-angle neutron scattering for extremely small sample volumes in the thin-film geometry. Although real space analysis techniques, such as atomic force microscopy, provide easy access to surface structures, reciprocal space analysis techniques, such as GISANS, provide several advantages: (i) average statistical information over the large illuminated sample surface can be detected and (ii) buried lateral structures can be probed without damage, using the variable-probed depth as a function of the incident angle. To illustrate the potential applications and challenges of GISANS, several different examples of thin nanostructured polymer films are reviewed. Nanostructures in triblock copolymer thin films are studied in the bulk as well as at the polymer-air and the silicon-polymer interface. Confined nanostructures in a dewetted diblock copolymer film are also discussed in terms of contrast and experimental settings.

Polymer Journal (2013) 45, 34-42; doi:10.1038/pj.2012.190; published online 14 November 2012

Keywords: block copolymer; dewetting; GISANS; polymer nanostructure; thin film

\section{INTRODUCTION}

Nanostructures and nanoparticles are of increasing importance in technological applications for many different scientific areas, such as energy (storage, transport and transformation), information technology and health. ${ }^{1-9}$ There is a need for sufficiently precise characterization methods to accompany improvements in the synthesis and preparation of these nanomaterials. Neutron scattering has proven to be a very powerful tool in the investigation of different classes of materials, such as biological, polymeric and magnetic materials, because of the particular interaction of the neutrons with these materials. ${ }^{10}$ The capability for selective deuteration in biological and polymer systems provides a fundamental advantage, as protonated and deuterated (bio) polymers can be easily distinguished in neutron scattering experiments because of the large difference in scattering crosssections between them. ${ }^{11}$ Moreover, biological and polymer materials are typically not susceptible to radiation damage when probed with neutrons, which is a problem typically encountered in experiments using intense synchrotron radiation.

Standard techniques, such as small-angle neutron scattering (SANS), for nanostructured sample volumes are very well established and have been widely applied. However, SANS experiments become difficult for investigating thin-film samples or nanostructures on solid supports because the scattering volume is simply too small in the transmission geometry. Stacking several (for example, 20) identically prepared samples (of nanostructures on solid support) has been shown to be necessary to improve the signal-to-noise ratio sufficiently to obtain a SANS signal. ${ }^{12}$ Replacing the transmission geometry by the reflection geometry overcomes the noise problems for cases where a very shallow incident angle is selected. This technique is known as grazing incidence diffraction. ${ }^{13}$ Combining the setup for the SANS measurements with the grazing incidence geometry produces a class of scattering experiments known as GISANS. ${ }^{14-20}$

Initial reports ${ }^{21}$ of grazing incidence experiments with X-rays (known as grazing incidence small-angle X-ray scattering (GISAXS)) date back to 1989, the first GISAXS experiments were performed on polymer samples in $1996 .^{22}$ However, the development of GISANS has been slower, as the first GISANS data was published in 1999. ${ }^{14,15}$ Meanwhile, GISANS has become well received as an advanced scattering technique. ${ }^{23-45}$ However, the number of reported GISANS experiments is still very limited compared with GISAXS, possibly because of continued restrictions on performing GISANS experiments compared with numerous GISAXS experiments at synchrotron radiation facilities. An interesting variation on the typical GISANS technique, known as time-of-flight GISANS (short TOF-GISANS), ${ }^{30,35,39}$ has recently been developed. TOF-GISANS has 
no well-established counterpart in the X-ray field to date because of the inherent problems with radiation damage in using X-rays.

In this review, recent studies by the Müller-Buschbaum group and their collaborators are used to illustrate the potential applications and challenges of GISANS, rather than attempting a broad overview of all the GISANS experiments for different sample systems in general. The examples are restricted to GISANS experiments using fixed neutron wavelengths and thus do not include TOF-GISANS. The review is structured as follows: first, a short introduction to the basics of the technique and the principles of the necessary setup is given; next, examples are presented on the detection of thin-film nanostructures and the investigation of the depth dependence of nanostructures via the variation of the incident angles; and finally, an investigation of confined nanostructures is presented, including the advantages of contrast enhancement and the importance of the setup selection.

\section{GISANS-BASIC PRINCIPLES}

In contrast to TOF-GISANS, GISANS is performed at a fixed neutron wavelength. As GISANS is always flux limited, the neutron wavelength producing the highest neutron flux should be used, as long as the wavelength is on the scale of the desired resolution of the nanostructures. Like in SANS, a 10\% wavelength resolution typically ensures sufficient neutron flux.

In the GISANS geometry, the neutron beam impinges onto the sample surface at a shallow incident angle $\alpha_{i}$, and the scattered intensity is detected at a different exit angle $\alpha_{f}$ and an out-of plane angle $\psi$. Figure 1 shows a basic sketch of this geometry, with a typical GISANS pattern containing the most commonly observed features of the specular peak (denoted by S), where the incident and exit angles are equal, and the so-called Yoneda peak (denoted by Y) ${ }^{46}$ where the exit angle is equal to the critical angle $\alpha_{c}$ of the material under investigation. ${ }^{13}$ If the coordinate system is chosen such that the sample surface defines the $(\mathrm{x}, \mathrm{y})$ plane where the neutron beam is directed in the $\mathrm{x}$-direction, the scattering plane is defined by the $(\mathrm{x}, \mathrm{z})$

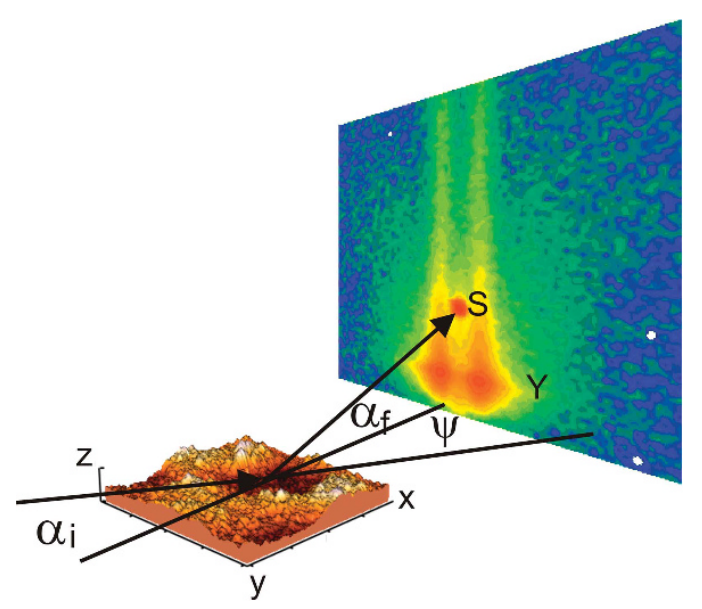

Figure 1 Schematic of the scattering geometry used in GISANS experiments. The sample is placed in the $(x, y)$ plane, and the incident neutron beam is along the $x$ axis. The incident angle of the neutron beam with respect to the sample surface is denoted by $\alpha_{i}$, the exit angle relative to the $\mathrm{x}$ axis in the $\mathrm{z}$ direction is denoted by $\alpha_{f}$, and the out-of plane angle relative to the $\mathrm{x}$ axis in $\mathrm{y}$ direction is denoted by $\psi$. The resulting scattering pattern is anisotropic and typically exhibits a Yoneda peak (marked with $Y$ ) and a specular peak (marked with S), which are described in the text. plane. ${ }^{16}$ For neutrons of wavelength $\lambda$, the corresponding scattering vector $\mathbf{q}=\left(\mathrm{q}_{x}, \mathrm{q}_{p}, \mathrm{q}_{z}\right)$ has the components

$\mathrm{q}_{x}=2 \pi\left(\cos \psi \cos \alpha_{f}-\cos \alpha_{i}\right) / \lambda$

$\mathrm{q}_{y}=2 \pi\left(\sin \psi \cos \alpha_{\mathrm{f}}\right) / \lambda$

$\mathrm{q}_{z}=2 \pi\left(\sin \alpha_{i}+\sin \alpha_{f}\right) / \lambda$.

Therefore, precise control of the angles $\alpha_{i}$ and $\psi$ is necessary for both the alignment and measurement of the thin-film samples. The $\mathrm{q}_{y}$ component, which is oriented parallel to the sample surface, probes lateral structures in a GISANS experiment. ${ }^{14-19}$ Consequently, the $\mathrm{q}_{y}$ resolution is a key prerequisite of GISANS. ${ }^{16}$ Lack of $\mathrm{q}_{y}$ resolution results in so-called conventional diffuse or off-specular scattering that describes only the $\left(\mathrm{q}_{x}, \mathrm{q}_{z}\right)$ plane. ${ }^{47-50}$ To achieve the $\mathrm{q}_{\mathrm{y}}$ resolution necessary for GISANS experiments, a point-shaped neutron beam source must be used instead of the slab-shaped beam source used in conventional diffuse scattering or reflectivity experiments. ${ }^{16}$ The point-shaped beam source can be installed by using additional slits in the collimation section of the experimental setup, similar to the SANS experimental setup. ${ }^{14,15}$ Typical slit sizes range from 1 to $10 \mathrm{~mm}$ (depending on the collimation and resolution required). Therefore, GISANS experiments are commonly conducted using SANS instruments rather than instruments that have been optimized for neutron reflectivity. ${ }^{14,15,35-37}$ Beam guides in the collimation section of the apparatus are detrimental because they reduce the resolution.

The sample is placed on a $(\mathrm{x}, \mathrm{y}, \mathrm{z})$ stage to control the sample position. As the neutron beam usually passes through the entire sample, a sample holder can avoid scattering parts of the neutron beam. Scattering from the sample holder adds to the background scattering, which can be higher in intensity than the actual GISANS signal. A two-circle goniometer (at minimum) is needed to control the incident angle and the tilt angle, with respect to the scattering plane. The tilt angle needs to be carefully aligned to facilitate accurate GISANS measurements. Pre-aligning the sample using a laser (instead of the neutron beam) is recommended. The incident angle is typically smaller than one degree and in the range of the critical angle of the investigated material. The incident angle is held fixed in GISANS. Overilluminating the sample at shallow incident angles reduces the intensity, increases the background scattering and should be avoided by using appropriate slit settings and large (long) samples. Evacuating the majority of the pathway in the entire setup also reduces background scattering, improving GISANS signal detection. The number of windows in the beampath should be minimized for the same reason. The scattered intensity can be most efficiently collected with a two-dimensional (2D) detector because GISANS scattering patterns are typically highly anisotropic; in addition, the scattered intensity is weak due to limited neutron flux, and thus, probing of the entire scattering pattern with one-dimensional or point detectors would require unrealistically long counting times. Diffusely scattered neutrons are best used with efficient detectors. Background scattering due to the detector should be minimized by using additional shielding against background scattering from other instruments. When the beam size is limited by the size of the detector pixels, the sampledetector distance sets the resolution in the GISANS setup. ${ }^{16}$ Only the direct beam, which passes through the sample and the sample holder in GISANS measurements, needs to be shielded with a beamstop to improve the background scattering from the detector. A beamstop is beneficial even when the detector can withstand the intensity of the direct beam. The GISANS signal is located above the sample horizon; thus unlike for GISAXS measurements, typically no additional beamstop is needed (for example, to block the specular peak where the incident and exit angles are equal). Consequently, the signal of 
interest, which is related to the resolution of large-scale structures at small values of $\mathrm{q}_{y}$, is not located in the vicinity of a beamstop. ${ }^{14-16}$ Thus, in the GISANS geometry, larger structures can typically be resolved compared with SANS experiments for the same collimation setting; as in SANS measurements, the larger structures scatter close to the shielded direct beam. ${ }^{29}$

Because GISANS measures diffusely scattered neutrons, GISANS signals are generally weak and require quite long counting times on the order of hours. ${ }^{14-20}$ Even with careful sample alignment, reasonably long counting times are expected as the Yoneda peak may not become visible within seconds of data acquisition.

An example of instrumental settings for GISANS are as follows: wavelength of $0.6 \mathrm{~nm}(\Delta \lambda / \lambda=10 \%), 17.6 \mathrm{~m}$ for both collimation and sample-detector distances, slit size of 10 and $1 \mathrm{~mm}$ in diameter and detector pixel size $0.8 \mathrm{~mm}$.

Data analysis is more complex for GISANS experiments than for SANS experiments. In addition to scattering from nanostructures, reflection at the substrate prior to and/or after the scattering event causes additional contributions to the diffuse scattering crosssection. ${ }^{23,45,51}$

A description of scattering based on a refractive index ${ }^{52}$ can be formulated, assuming that the atomic or molecular structure can be neglected because of the small angles involved in GISANS, where the scattering arises from variations in the scattering length density (SLD):

$$
n=1-\delta+i \beta
$$

where $\delta=N b \lambda^{2} / 2 \pi, \beta=N \alpha_{a} \lambda / 4 \pi, N$ is the atomic number density, $b$ is the coherent scattering amplitude of the bound atom (also called scattering length), $\alpha_{a}$ the absorption cross-section for neutrons and $N b$ is the neutron SLD. ${ }^{12}$ For X-rays, $\delta=r_{0} \rho_{e} \lambda^{2} / 2 \pi$ and $\beta=\mu \lambda / 4 \pi$, where $r_{0}$ is the electron radius, $\rho_{e}$ is the electron density of the medium and $\mu$ is the linear absorption coefficient.

In contrast to X-ray scattering lengths $r_{0} \rho_{e}$ that increase with the number of electrons of the investigated atoms, neutron scattering lengths vary randomly from element to element and from isotope to isotope. Isotopic substitution can be used to produce large differences in the SLD and refractive index. ${ }^{53}$ The scattering lengths of hydrogen and deuterium differ both in sign and magnitude, which leads to many practical applications in terms of contrast enhancement and contrast matching. ${ }^{11}$

A description within the framework of the distorted wave Born approximation, a first-order perturbation theory, ${ }^{47-50,51}$ is usually used to include strong dynamical effects from total external reflection. The diffuse scattering cross-section of an $m$-layer system is ${ }^{48-50}$

$$
\left(\frac{d \sigma}{d \Omega}\right)_{\text {diff }}=\frac{C \pi^{2}}{\lambda^{4}} \sum_{j, k=1}^{m}\left(n_{j}^{2}-n_{j+1}^{2}\right)\left(n_{k}^{2}-n_{k+1}^{2}\right) \sum_{h, l=0}^{3} W_{h, j} W_{l, k} F_{j, k}^{h, l}
$$

with the Fresnel transmissions coefficients $T_{i, f}$ and the Fresnel reflection coefficients $R_{f, j}$ entering directly in $W_{0, j}=T_{i, j} T_{f, j}$; $W_{1, j}=T_{i, j} R_{f, j} ; \quad W_{2, j}=R_{i, j} T_{f, j} \quad$ and $\quad W_{3, j}=R_{i, j} R_{f, j} . \quad C$ denotes the illuminated surface area and $n_{j}$ denotes the refractive index of the jth-layer. The data cannot be fitted in a straightforward manner because of the q-dependence of the diffuse scattering factor $F_{j, k}^{h, l}$.

Lateral structures can be investigated by taking so-called horizontal line cuts at a fixed exit angle, where the intensity is simply plotted as a function of $\mathrm{q}_{\mathrm{y}}$.

Interface sensitivity can be achieved by varying the incident angle in GISANS experiments, as is well known from grazing incidence diffraction experiments. ${ }^{13}$ At incident angles smaller than the critical angle,

$$
\alpha_{c}=\lambda \sqrt{\frac{N b}{\pi}},
$$

the transmitted wave is exponentially damped into the less dense medium, limiting the penetration of the beam into the sample. Both the selected incident and exit angles, $\alpha_{i}$ and $\alpha_{f}$ must be accounted for to determine the depth from which the observed scattering originates. The scattering depth of the neutrons

$$
D=\frac{\lambda}{\sqrt{2} \pi\left(l_{i}+l_{f}\right)}
$$

depends both on the grazing angles $\alpha_{i}$ and $\alpha_{f}$ and the difference in the SLDs via ${ }^{13}$

$$
l_{i, f}=\left[\left(\alpha_{c}^{2}-\alpha_{i, f}^{2}\right)+\sqrt{\left(\alpha_{i, f}^{2}-\alpha_{c}^{2}\right)^{2}+4 \beta^{2}}\right]^{1 / 2} .
$$

$D$ represents the distance from the polymer-air surface when the neutron beam enters the polymer film from that surface. ${ }^{29}$ When the neutron beam enters through a transparent substrate, such as silicon
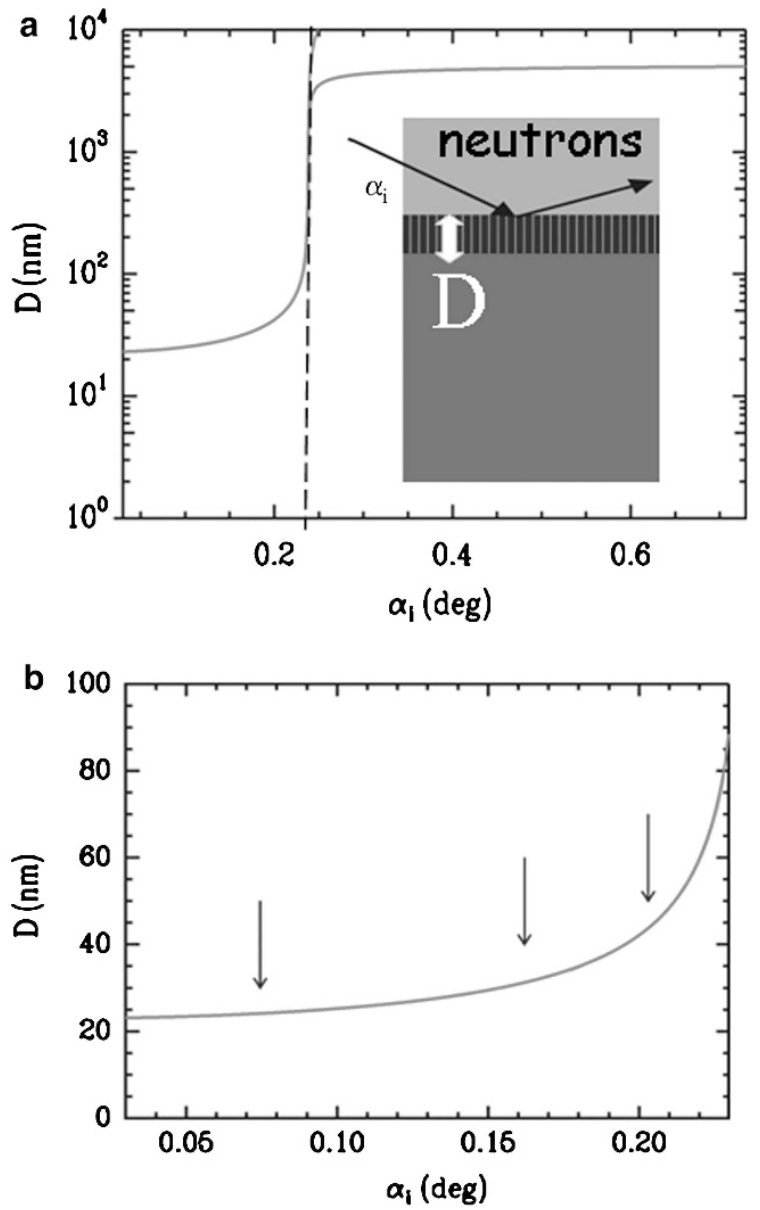

Figure 2 Scattering depth $D$ as a function of the incident angle $\alpha_{i}$, for (a) $\alpha_{i}$ smaller than and greater than $\alpha_{c}$ (the dashed line indicates $\alpha_{c}$ ) and (b) $\alpha_{i}<\alpha_{c}$ (selected values of incident angles of $0.075,0.162$ and $0.213^{\circ}$ are marked with arrows). The inset in a shows a sketch of the scattering geometry for neutrons entering the polymer film through a transparent substrate such as Si (depicted in gray). A full color version of this figure is available at Polymer Journal online. 
(Si), $D$ is the distance from the $\mathrm{Si}$-polymer interface into the polymer (as depicted in the inset of Figure 2). ${ }^{33}$

The highly nonlinear behavior of $D\left(\alpha_{i}\right)$ is illustrated in Figure 2 for the polymer film under investigation. The probed depth changes significantly for an incident angle close to the critical angle $\alpha_{c}$ For $\alpha_{i}<\alpha_{c}$, surface or interface sensitivity is achieved using a very limited scattering depth.

\section{DETECTION OF THIN-FILM NANOSTRUCTURES}

To illustrate how GISANS can probe polymer nanostructures, experiments were conducted on the internal nanostructure resulting from micro-phase separation in triblock copolymer thin films of polyparamethylstyrene (PpMS)-block-polystyrene (PS) (deuterated)block-PpMS, P(pMS- $b$-Sd- $b$-pMS) with a molecular mass $M_{w}=$ $280000 \mathrm{~g} \mathrm{~mol}^{-1}$ and a narrow molecular weight distribution $M_{w} / M_{n}=1.1 .^{29}$ The degree of polymerization of the PSd block, compared with the total chain, was $f_{\text {PSd }}=N_{\text {PSd }} / N=0.51$. The influence of two limiting interfaces in thin films, which impose a spatial restriction of the polymer, was compared with the presence of only one surface in bulk-like films. $\mathrm{P}$ (pMS- $b$-Sd- $b$-pMS) films of different thicknesses $\left(d_{\text {sum }}=0.7,27.0,71.8\right.$ and $\left.230.8 \mathrm{~nm}\right)$ were investigated with GISANS using a neutron wavelength of $0.6 \mathrm{~nm}$ (Figure 3). ${ }^{29}$ The discussion is restricted to selected horizontal line cuts $^{16}$ instead of considering the full $2 \mathrm{D}$ intensity distributions. The relevant cuts were performed at exit angles $\alpha_{f}=\alpha_{c}$ (PpMS) $<\alpha_{c}(\mathrm{Si})$, corresponding to exit angles smaller than the PSd critical angle, $\alpha_{c}$ (PSd). All horizontal cuts exhibit intensity maxima well inside the range of resolvable lateral lengths, independent of the film thickness (Figure $3 \mathrm{a}$ ). There is a very pronounced intensity maximum for the 230.8-nm thick bulk-like P(pMS- $b$-Sd- $b$-pMS) film. The strength of the maximum decreases with decreasing film thickness. However, a weak but clearly visible peak is observed above the background level even for a 0.7-nm film thickness. To achieve this level of statistical detail in the GISANS data, a 8-h counting time was necessary even with a beamline with a very high neutron flux (D22; ILL, Grenoble, France). ${ }^{29}$ In addition to the change in intensity with decreasing film thickness, the position of the intensity maximum shifts toward larger values of the wave vector component $\mathrm{q}_{\mathrm{y}}$. This shift resembles the shrinkage of the most prominent in-plane length of the ordered triblock copolymer. Owing to the absence of real space information and higher-order intensity maxima in the horizontal cuts, we interpret this shrinkage as a decrease in the lamellar spacing $L_{0}$, rather than a morphological transition due to confinement. Such a transition, for example, from a lamellar to a cylindrical structure, would have introduced a change in the most prominent in-plane length as well. ${ }^{29}$

$L_{0}$ was extracted from fitting the horizontal line cuts in the distorted wave Born approximation framework. ${ }^{47,29}$ To emphasize confinement-induced shrinkage, $L_{0}$ is normalized by the value of the bulk lamellar spacing $L_{\text {bulk }}$ (determined from transmission experiments), which is a function of the total triblock copolymer film thickness $d_{\text {sum }}$ (Figure $3 \mathrm{~b}$ ). The solid line is drawn as a guide for the eye. Obviously, more than $30 \%$ shrinkage in the lamellar spacing only occurs in the strongest confinement cases. ${ }^{29}$

\section{DEPTH DEPENDENCE OF NANOSTRUCTURES}

Figure $2 \mathrm{~b}$ shows that slight variations in the incident angle can finely tune the scattering depth with nanometer precision inside the boundaries imposed by the sample system (SLD difference) and the experimental setup (the precision given for $\alpha_{i}$ and the wavelength distribution as given by $\Delta \lambda / \lambda$ ). For $\alpha_{i}>\alpha_{c}$, GISANS becomes sensitive to the bulk, resulting in the detection of structures in the entire thin film. The maximum scattering depth is mainly determined by the absorption cross-section of the neutrons in the probed polymer film. When the incident angle is smaller than the critical angle, the probed polymer volume is restricted by the scattering depth $D$ to regions near the interface. For example, incident angles of $0.075,0.162$ and $0.213^{\circ}$ at the triblock copolymer-Si interface (marked with arrows in Figure 2b) produce scattering depths of 24,31 and $51 \mathrm{~nm}$, respectively. ${ }^{33}$

\section{Nanostructures at the free surface of the polymer film}

A series of GISANS experiments were conducted to investigate deviations from $L_{\text {bulk }}$ at the surface of the triblock copolymer film $\mathrm{P}$ (pMSb-Sd-b-pMS). A neutron beam (wavelength $0.6 \mathrm{~nm}$ ) entered the polymer from the polymer-air surface at different incident angles:
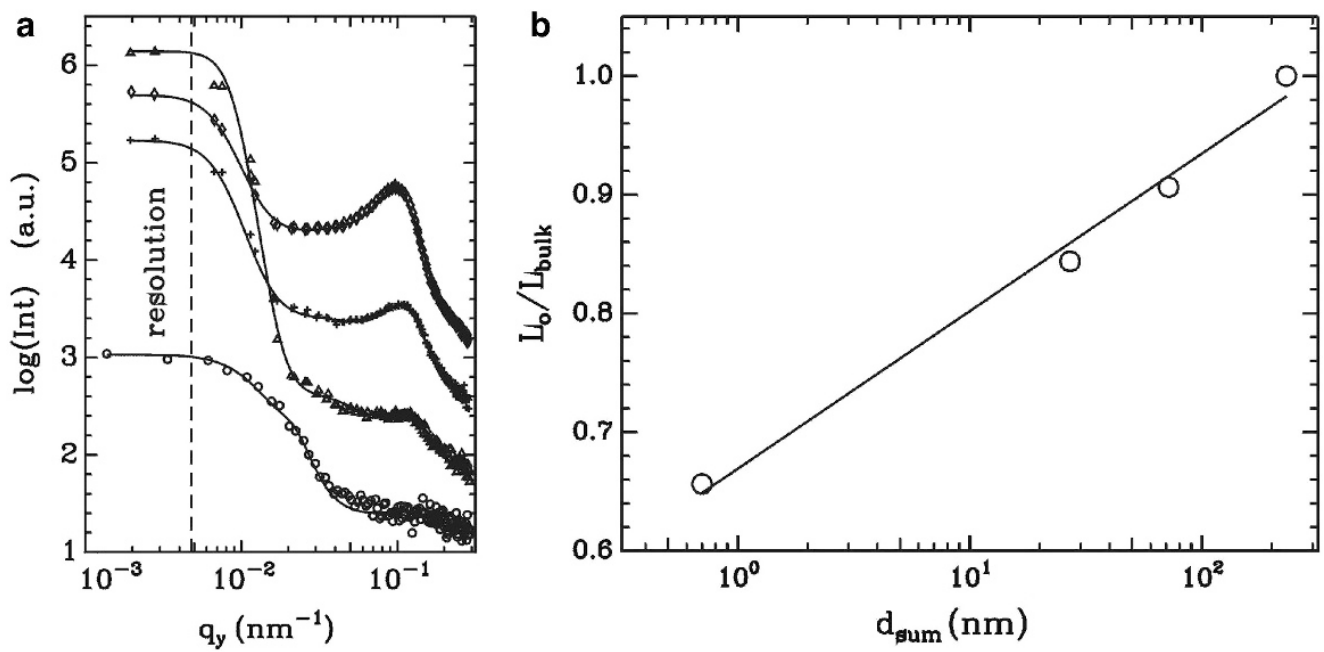

Figure 3 (a) Horizontal line cuts from 2D GISANS data, as described in the text. The prepared P(pMS- $b$-Sd- $b$-pMS) film thickness increases from bottom to top $\left(d_{\text {sum }}=0.7,27.0,71.8\right.$ and $230.8 \mathrm{~nm}$ ). The solid lines are fits to the data within the distorted wave Born approximation framework. The dashed line indicates the resolution limit. All curves are shifted along the y axis for clarity. (b) Lamellar spacing $L_{0}$ normalized by the bulk value $L_{\text {bulk }}$ as a function of the total triblock copolymer film thickness $d_{\text {sum. }}$. The solid line is shown to guide the eye. Reprinted with permission from Langmuir 22, 9295-9303 (2006). Copyright (2006) American Chemical Society. 

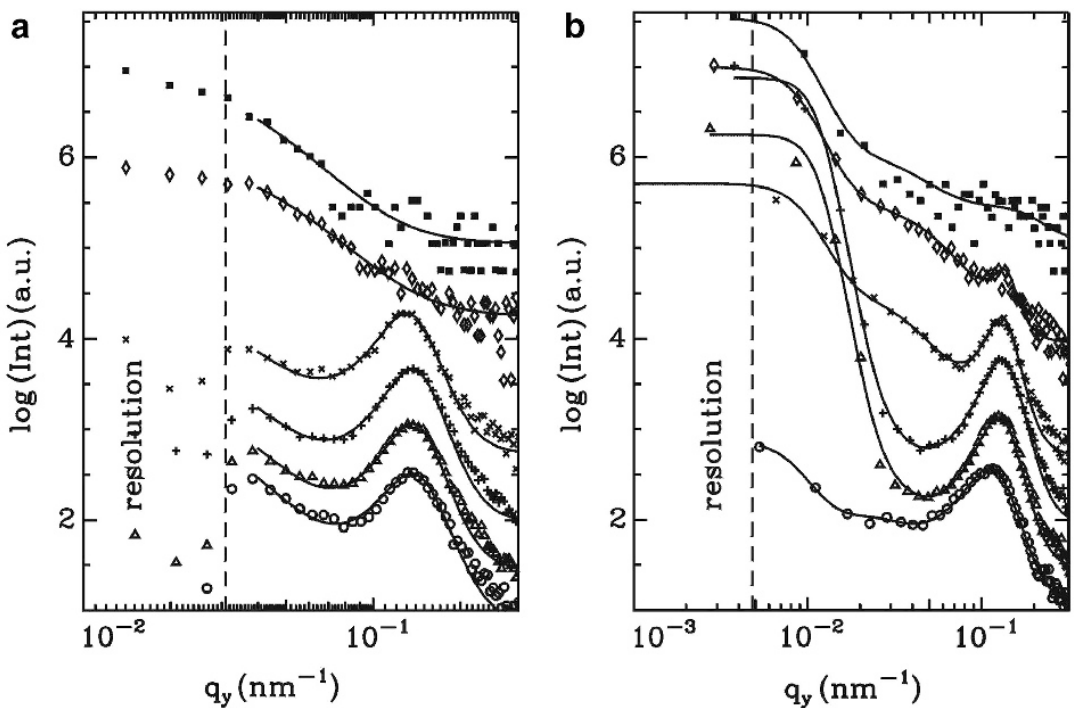

Figure 4 Horizontal line cuts from 2D GISANS data of a 230.8-nm thick P(pMS- $b$-Sd- $b$-pMS) film (a) at the central position of the beamstop and (b) at the critical angle. The incident angle increases from top to bottom $\left(\alpha_{i}=0.067,0.162,0.306,0.417,0.465\right.$ and $\left.0.719^{\circ}\right)$. The solid lines are model fits. The dashed line indicates the resolution limit. All curves are shifted along the y axis for clarity. Reprinted with permission from Langmuir 22, 9295-9303 (2006). Copyright (2006) American Chemical Society.

$\alpha_{i}=0.067,0.162,0.306,0.417,0.465$ and $0.719^{\circ} .^{29}$ The material investigated was again $\mathrm{P}$ (pMS- $b$-Sd- $b$-pMS), with a molecular mass $M_{w}=280000 \mathrm{~g} \mathrm{~mol}^{-1}$, a narrow molecular weight distribution $M_{w} / M_{n}=1.1$ and $f_{\mathrm{PSd}}=N_{\mathrm{PSd}} / N=0.51$.

The two lowest incident angles, $\alpha_{i}=0.067$ and $0.162^{\circ}$, are both smaller than the critical angles of the materials under investigation. $\alpha_{i}=0.306^{\circ}$ is smaller than the critical angles for the mean bulk $\mathrm{P}$ (pMS- $b$-Sd- $b$-pMS) and PSd, but greater than the critical angles for PpMS and Si. The incident angle $\alpha_{i}=0.417^{\circ}$ is only smaller than the critical angle of PSd, and all larger incident angles are larger than all critical angles under consideration. Two different horizontal line cuts are chosen for the analysis: one cut is at the center of the beamstop (Figure 4a) and the other is at the PpMS critical angle (Figure 4b). A horizontal cut at the center of the beamstop corresponds to $\mathrm{q}_{\mathrm{z}}=0$, showing that the transmitted neutron intensity is free of refraction effects, as refraction only occurs in the $\mathrm{q}_{\mathrm{z}}$ direction. $L_{\text {bulk }}$ was determined from fitting the data. For very shallow incident angles, the neutron beam cannot penetrate the triblock copolymer film to produce a transmission signal. As a consequence, for the two incident angles smaller than all critical angles of the related materials (PpMS, Si and PSd), no intensity maximum due to the internal micro-phase separation structure was observed. ${ }^{29}$

Horizontal line cuts at the critical angle are composed of contributions from surface scattering and bulk scattering, giving rise to strong intensity maxima (Figure $4 \mathrm{~b}$ ). The position of these maxima changes with the incident angle because of refraction. The shift due to variations in the incident angle should not be confused with a change in the lamella spacing of the micro-phase-separated structure. The intensity of the maximum arising from bulk-like scattering increases with decreasing incident angles (for incident angles greater than the critical angle of the materials) because an increasing polymer film volume is being probed due to the projection of the neutron beam. The intensity maximum stops increasing at the angle for which the neutron beam can no longer penetrate the film due to total external reflection. Consequently, for incident angles equal to or smaller than the critical angle of the materials, the horizontal line cuts contain only structural information about the surface, and there is no bulk scattering signal. The probed polymer volume significantly decreases, which explains the reduced intensity. Moreover, the increase in the footprint with decreasing incident angle typically reduces the scattered intensity due to the limited sample size. The presence of intensity maxima in these horizontal line cuts can only be explained by the existence of a well-ordered lamellar structure that is oriented perpendicularly with respect to the surface. The related periodicity $L_{\text {surface }}$ is extracted from the distorted wave Born approximationbased fit. $L_{\text {surface }}$ shows $5 \%$ stretching of the chains compared with $L_{\text {bulk. }}{ }^{29}$

\section{Nanostructures at a buried interface}

Owing to the unique abilities of neutrons to penetrate many of the substrates commonly used for thin polymer films (e.g., Si, glass, and titania), lateral structures at buried interfaces can also be probed. As mentioned above, the neutron beam needs to enter through the substrate in these experiments. ${ }^{33}$ As for the structures at the free surface of polymer films, depth sensitivity is achieved by varying the incident angle (see Figure 2b). Critical angles for the polymersubstrate interface are typically smaller than critical angles for the free surface of the films because the SLD difference between the polymer film and the substrate is smaller than that between polymer and air $(\operatorname{SLD}($ air $)=0){ }^{12}$

To illustrate the influence of different substrate surface treatments on lateral structures, $\mathrm{P}$ (pMS- $b$-Sd- $b$-pMS) films were probed with GISANS (wavelength $0.6 \mathrm{~nm}) .^{33} \mathrm{P}(\mathrm{pMS}-b$-Sd- $b$-pMS) with a molecular mass $M_{w}=280000 \mathrm{~g} / \mathrm{mol}\left(M_{w} / M_{n}=1.1\right.$ and $\mathrm{f}_{\mathrm{PSd}}=\mathrm{N}_{\text {PSd }} /$ $\mathrm{N}=0.51$ ) was investigated. A perpendicular orientation of the lamellae of the micro-phase-separated structure of the triblock copolymer was found, independent of the surface treatment of the Si substrate (acid cleaning, base cleaning, deposited PDMS grains and on a PS brush surface). The presence of an intensity maximum in the horizontal line cuts of the 2D GISANS data is clear proof of the perpendicular orientation. ${ }^{33}$ The absence of intensity maxima along the $\mathrm{q}_{\mathrm{z}}$ direction is further proof of the absence of a lamellar 
orientation parallel to the substrate surface, as shown in MüllerBuschbaum et l. $^{33}$

To determine the extent of order with the distance from the interface, using GISANS in triblock polymer films for different substrate interfaces, different incident angles were chosen relative to the critical angle $\alpha_{c}$ of the copolymer film in contact with Si: $\alpha_{i} / \alpha_{c}=0.313,0.675,0.888$, and 3.029 with $\alpha_{c}=0.24^{\circ}$. Thus, three incident angles smaller than the critical angle of the triblock copolymer in contact with the Si substrate were selected, and one angle was selected that was larger than the critical angle. ${ }^{33}$

Figure 5 shows measurements of the increase in lamellar spacing near the SiOx substrate for the different surface treatments from the GISANS data analysis. For the base cleaned sample, no deviation of the lamellar spacing from the bulk spacing was found at the buried interface. In contrast, for the acid cleaned sample, the PDMS grains and the PS brush, the lamellar spacing near the interface was larger than the spacing in the bulk. The degree of chain stretching decreased with increasing distance from the substrate until the bulk value was reached. Chains stretch less with increasing distance from the substrate due to the reduction in the substrate enthalpic contribution. However, the maximum stretch and the decay in chain stretching depend on the surface treatment, which is discussed in detail in terms of a neutral, attractive or repulsive wall in Müller-Buschbaum et al. ${ }^{33}$

\section{DETECTION OF CONFINED NANOSTRUCTURES}

The detection of polymer nanostructures is a particular strength of GISANS, especially for nanostructures of polymers with very similar SLDs for X-ray measurements. ${ }^{14-16}$ A study of polymer nanodewetting structures is reviewed as an illustration. ${ }^{18}$ An initially ultra-thin polymer film, with a film thickness smaller than the radius of gyration of the polymer, dewets into isolated polymer droplets on a solid support. Such dewetting structures have been reported for homopolymer systems, polymer blends and diblock copolymers. Examples are PS on $\mathrm{Si}^{14,15}$ PS blended with PpMS on $\mathrm{Si}^{15-17}$ and

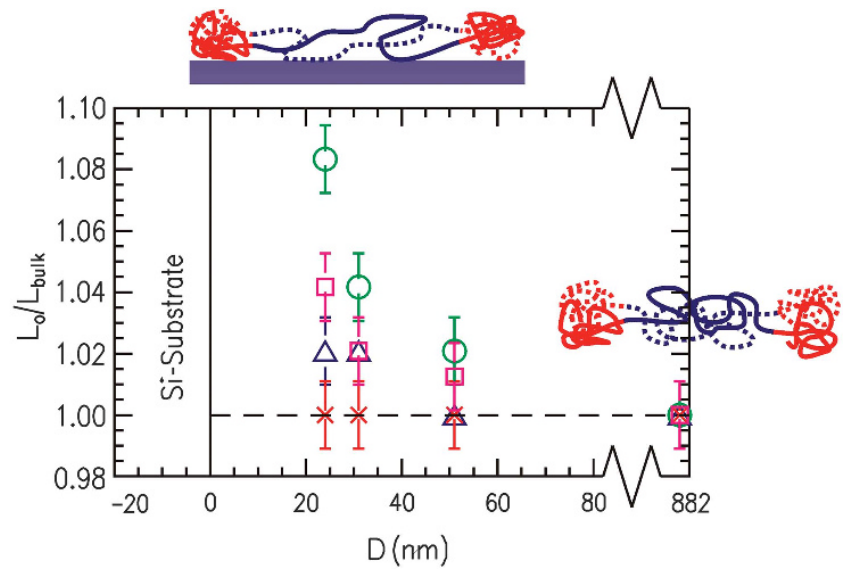

Figure 5 Lamellar spacing $L_{0}$ normalized by the bulk lamellar spacing $L_{\text {bulk }}$ probed as a function of the scattering depth $D$ of the signal (that is, the distance over which the signal is probed with the evanescent wave from the substrate-polymer interface) for the triblock copolymer P(pMS- $b$-Sd- $b$-pMS) deposited on a Si substrate after acid cleaning (triangles) and base cleaning (crosses), with deposited PDMS grains (squares) and on the PS brush surface (spheres). The dashed line shows $L_{\text {bulk }}$ of the micro-phase-separated triblock copolymer. The SiOx substrate is shown. The sketched polymer chains illustrate chain stretching close to the substrate. Partially reproduced from Langmuir 24, 7639-7644 (2008) with permission. Copyright (2008) American Chemical Society.
PS-block-polyparamethlystyrene (P(S- $b$-pMS)) on Si. ${ }^{18,54}$ In all cases, droplets are formed with a pancake shape. Thus, confinement is imposed by the very limited droplet volume. The preferred nearestneighbor distance between droplets is shown in Figure 6a. The surface topography can be probed with real space techniques, such as atomic force microscopy (AFM). However, AFM cannot provide information about the inner nanostructure of these droplets if no further contrast, such as mechanical contrast, is present between the components of a blend or a block copolymer. ${ }^{14-16}$

Figure 6 shows the determination of a droplet structure for $\mathrm{P}(\mathrm{Sd}-b$ pMS) with a deuterated PS block (PSd) and a protonated PpMS block (molecular weight $M_{w}=230000 \mathrm{~g} \mathrm{~mol}^{-1}$, narrow molecular weight distribution of $\left.M_{w} / M_{n}=1.08\right) .{ }^{54}$ The droplet structure is well elucidated with AFM for several different scan sizes and at different positions on the sample, but the statistics are more limited than those obtained by GISANS (or GISAXS). ${ }^{18}$ However, lacking mechanical or chemical contrast between PSd and PpMS (where PSd and PpMS differ by only one methyl group, despite deuteration), ${ }^{17}$ AFM cannot access the inner nanostructure of the droplets. Therefore, GISAXS and GISANS measurements were performed to investigate the inner structure. GISAXS was performed at beamline BW4 at HASYLAB in Hamburg (Germany), and GISANS was conducted at beamline D22 at ILL (wavelength $0.6 \mathrm{~nm}$, incident angle $\alpha_{i}=0.645^{\circ}$ ). Figure 6b compares the master curve calculated from the power spectral density data of individual-sized AFM images with a horizontal line cut from 2D GISAXS and GISANS data taken at the critical angle of the investigated material. A well-defined inter-droplet distance gives rise to an intensity maximum in the AFM master curve (denoted as I in Figure $6 \mathrm{~b}$ ), whereas the form factor of the droplets contributes on a smaller-length scale (denoted as II in Figure 6b). In the GISAXS and GISANS experiments, the resolution is deliberately set to focus on smaller-length scales, such as inner structures, leaving the dropletdroplet distance unresolved. Therefore, the form factor is observed close to the resolution limit (indicated by the dashed line in Figure 6b). An additional peak (denoted as III in Figure 6b) is observed in the GISANS data, which is not found in the AFM master curve or the horizontal line cut of the GISAXS data. With X-rays, both blocks of the diblock copolymer are barely distinguishable $(\delta(P S d) / \delta(P p M S)=0.9)$, whereas with GISANS, sufficient contrast between both blocks is present $(\delta(P S d) / \delta(P p M S)=4.1) .{ }^{54}$ Figure 6c shows that GISANS can probe the inner chemical nanostructure originating from the micro-phase separation of the diblock copolymer $\mathrm{P}(\mathrm{Sd}-b$-pMS). The micro-phase-separated structure $(72 \mathrm{~nm})$ is oriented perpendicular to the substrate surface, as evidenced by a signal in the $\mathrm{q}_{\mathrm{y}}$ direction.

The detection of such nanostructures is challenging because of the extremely small available scattering volume. The ability to detect these nanostructures demonstrates the sensitivity of GISANS, but suitable experimental settings must be selected carefully. Figure 7 shows the influence of different experimental settings on the observed GISANS signal for a dewetted $\mathrm{P}\left(\mathrm{Sd}-b\right.$-pMS) film. ${ }^{54}$ The effect of changing the wavelength is shown for fixed slit settings, a collimation distance of $17.66 \mathrm{~m}$ and a sample-detector distance of $17.66 \mathrm{~m}$. GISANS measurements were performed with wavelengths of $0.6,1.0$ and $1.7 \mathrm{~nm}$. Horizontal line cuts were extracted from the 2D GISANS data at the critical angle of the material under investigation.

By increasing the neutron wavelength, the neutron flux is decreased as per the instrument characteristics (D22 at ILL) while simultaneously increasing the resolution and the coherently illuminated surface area. This decrease in neutron flux seriously affects the detectable GISANS signal, which can be observed by comparing the 

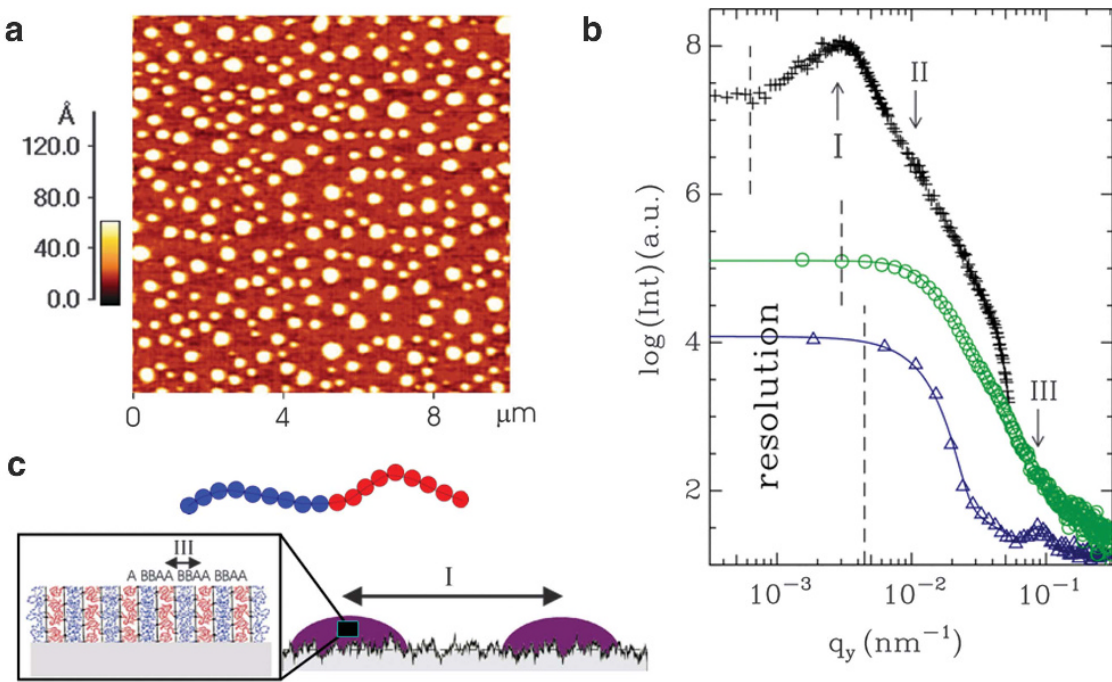

Figure 6 (a) AFM picture (scan range $10 \times 10 \mu \mathrm{m}^{2}$ ) of the topology signal showing the droplet structure of a P(Sd-b-pMS) diblock copolymer film on a Si substrate as observed after sample storage under toluene vapor for $12 \mathrm{~h}$. (b) Comparison between the master curve calculated from AFM data (crosses, top) and the horizontal line cuts of the GISAXS (circles, middle) and GISANS (triangles, bottom) data. The corresponding initial film thickness for the case shown is $6 \mathrm{~nm}$. Typical length scales are indicated by arrows and labeled I, II and III, as described in the text. The solid lines are fits to the GISAXS and to the GISANS data. The dashed lines depict the resolution limit, which is different for the three experimental techniques. In this double logarithmic presentation, all curves are shifted along the $y$ axis for clarity. (c) Sketch of the inner morphology of the polymer droplets. Partially reproduced from Langmuir 17, 5567-5575 (2001) with permission. Copyright (2001) American Chemical Society.

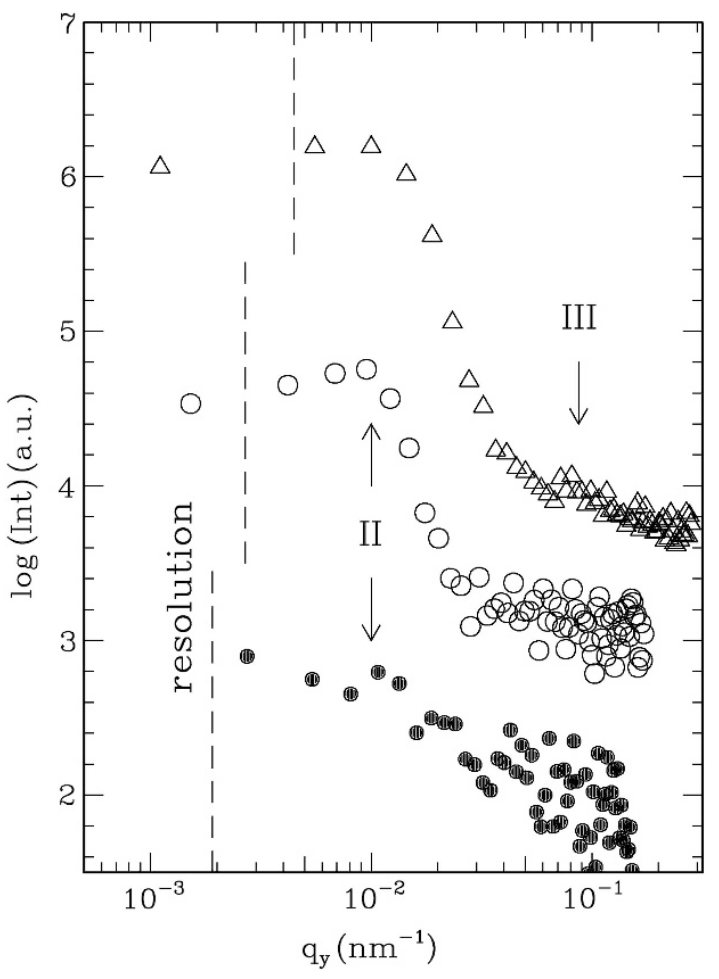

Figure 7 Double logarithmic plot of horizontal line cuts from 2D GISANS data of a dewetted P(Sd-b-pMS) film with an initial film thickness of $3 \mathrm{~nm}$, measured with different neutron wavelengths of $0.6 \mathrm{~nm}$ (triangles), $1.0 \mathrm{~nm}$ (circles) and $1.7 \mathrm{~nm}$ (filled circles). The experimental resolution is indicated by the dashed lines. Typical peaks are present at positions marked with II and III. All curves are shifted along the y axis for clarity. Partially reproduced from Langmuir 17, 5567-5575 (2001) with permission. Copyright (2001) American Chemical Society. peak intensities for the three different wavelengths at position II (Figure 7). Although a peak at position II is clearly visible for the 0.6 and 1.0-nm wavelengths, the peak for the $1.7-\mathrm{nm}$ wavelength is extremely weak. Thus, GISANS experiments with a wavelength of 0.6 and $1.0 \mathrm{~nm}$ are well suited, from intensity considerations, to probe structures that produce the peak at position II. However, the limit of resolution for a 0.6-nm wavelength is close to the scale of the dominant lateral structure, producing the position II peak. Thus, resolution limitations must be carefully accounted for during GISANS data analysis. As smaller lateral structures appear at larger $\mathrm{q}_{\mathrm{y}}$ values (for example, at position III), the corresponding intensity maximum is even weaker. As a consequence, peak III shown in Figure 7 is only weakly present for the measurement with the 0.6-nm wavelength and vanishes into the background noise for the measurements with the 1.0 and $1.7-\mathrm{nm}$ wavelengths. Typical accumulation times were longer than $6 \mathrm{~h}$; thus, the peak statistics are unlikely to improve by increasing the accumulation time. Therefore, a GISANS experiment with a wavelength of $0.6 \mathrm{~nm}$ is the best compromise to probe both the lateral structures (II and III). These results elucidate the limits of the GISANS method even for experiments performed at a beamline with high flux. ${ }^{54}$

\section{SUMMARY}

In summary, GISANS is an advanced scattering technique that involves a combination of two techniques: grazing incidence diffraction, which uses reflection geometry to obtain surface and nearsurface-sensitive scattering, and SANS, which measures structures in the 1-100-nm range in normal transmission mode. The advantages of GISANS include the following: GISANS is a non-destructive structural probe (that is, no radiation damage occurs) that yields excellent sampling statistics; and due to a large neutron beam size and sample footprint, GISANS can provide information on the nanometer scale by averaging over macroscopic regions. GISANS provides information on nanoparticle geometry, size distributions and spatial correlations 
of nanostructures, without the need for special sample preparation. Therefore, in situ characterization using GISANS is possible in principle. Depth sensitivity can be achieved by changing the incident angle, and buried interfaces such as a polymer-solid contact can be probed. In this focus review, examples of polymer nanostructures were used to illustrate the capabilities of GISANS. GISANS provides access to nanostructures that cannot be probed with GISAXS, especially for materials that have a low contrast for X-rays but a high contrast for neutrons. In addition to the growing number of applications of GISANS for polymer samples, GISANS can also be successfully applied to other materials that are of interest in condensed matter science.

However, currently, GISANS experiments remain flux limited owing to counting times on the order of several hours, which render these experiments challenging for typical instrumental settings. In addition, GISANS data analysis is more complex compared with SANS data analysis because of the reflection effects from the substrate surface. Typically, a distorted wave Born approximation-based approach is used for data analysis, which assumes a model for the nanostructure. In terms of the modeling required, direct comparison with other experimental data, for example, from AFM, is beneficial for constructing an appropriate model.

With new upcoming neutron sources, GISANS will grow in popularity in the future. The development of new variations of the GISANS technique, such as TOF-GISANS, will also contribute to the increasing number of GISANS experiments.

\section{ACKNOWLEDGEMENTS}

This work has benefited strongly from fruitful and enjoyable collaborations with E Bauer, R Cubitt, E Maurer, E Metwalli, J-F Moulin, W Petry, L Schulz, M Stamm, M Wolkenhauer and O Wunnicke and from discussions with R Campbell, R Pynn and M Rawolle.

1 Russell, T. P. Surface-responsive materials. Science 297, 964-967 (2002).

2 Kadota, S., Aoki, K., Nagano, S. \& Seki, T. Photocontrolled microphase separation of block copolymers in two dimensions. J. Am. Chem. Soc. 127, 8266-8267 (2005).

3 Tzanetos, N. P., Dracopoulos, V., Kallitsis, J. K. \& Deimede, V. A. Morphological study of the organization behavior of rod-coil copolymers and their blends in thin solid films. Langmuir 21, 9339-9345 (2005).

4 Ludwigs, S., Schmidt, K. \& Krausch, G. One-dimensional swelling of a pH-dependent nanostructure based on ABC triblock terpolymers. Macromolecules 38, 2376-2382 (2005).

5 Castro, F. A., Benmansour, H., Graeff, C. F. O., Nuesch, F., Tutis, E. \& Hany, R. Nanostructured organic layers via polymer demixing for interface-enhanced photovoltaic cells. Chem. Mater. 18, 5504-5509 (2006).

6 Fujikawa, S., Takaki, R. \& Kunitake, T. Fabrication of arrays of sub-20-nm silica walls via photolithography and solution-based molecular coating. Langmuir 22, 9057-9061 (2006).

7 Zhang, Y., Lo, C. -W., Taylor, J. A. \& Yang, S. Replica molding of high-aspect-ratio polymeric nanopillar arrays with high fidelity. Langmuir 22, 8595-8601 (2006).

8 Spontak, R. J., Shankar, R., Bowman, M. K., Krishnan, A. S., Hamersky, M. W., Samseth, J., Bockstaller, M. R. \& Rasmussen, K. O. Selectivity- and size-induced segregation of molecular and nanoscale species in microphase-ordered triblock copolymers. Nano Lett. 6, 2115-2120 (2006).

9 Müller-Buschbaum, P., Bauer, E., Maurer, E., Schlögl, K., Roth, S. V. \& Gehrke, R. A new route to large-area ordered polymeric nano-channel arrays. Appl. Phys. Lett. 88, 083114 (2006).

10 Higgins, J. S. \& Benoît, H. C. Polymers and neutron scattering (Clarendon Press, Oxford, 1994).

11 Stamm, M. \& Schubert, D. W. Interfaces between incompatible interfaces. Annu. Rev. Mater. Sci. 25, 325-356 (1995).

12 Jones, R. L., Kumar, S. K., Ho, D. L., Briber, R. M. \& Russell, T. P. Chain conformation in ultrathin polymer films. Nature 400, 146 (1999).

13 Dosch, H. Critical phenomena at surfaces and interfaces: Evanescent X-ray and neutron scattering (Springer-Verlag, Berlin, 1992)

14 Müller-Buschbaum, P., Gutmann, J. S. \& Stamm, M. Dewetting of confined polymer films: An X-ray and neutron scattering study. Phys. Chem. Chem. Phys. 1, 3857-3863 (1999).
15 Müller-Buschbaum, P., Gutmann, J. S., Cubitt, R. \& Stamm, M. Probing the in-plane composition of thin polymer films with grazing-incidence small angle scattering and atomic force microscopy. Colloid. Polym. Sci. 277, 1193-1199 (1999).

16 Müller-Buschbaum, P., Gutmann, J. S., Stamm, M., Cubitt, R., Cunis, S., von Krosigk, G., Gehrke, R. \& Petry, W. Dewetting of thin polymer blend films: examined with GISAS. Physica B 283, 53-59 (2000).

17 Müller-Buschbaum, P., Cubitt, R. \& Petry, W. Phase separation of weakly incompatible polymer blends confined into isolated droplets. App/ Phys. A 74, S342-S344 (2002).

18 Müller-Buschbaum, P. Dewetting and pattern formation in thin polymer films as investigated in real and reciprocal space. J. Phys. Condens. Matter 15, R1549-R1582 (2003).

19 Müller-Buschbaum, P., Cubitt, R. \& Petry, W. Nano-structured diblock copolymer films: A grazing incidence small-angle neutron scattering study. Langmuir 19, 7778-7782 (2003).

20 Steitz, R., Müller-Buschbaum, P., Schemmel, S., Cubitt, R. \& Findenegg, G. H. Lateral structure of a surfactant adsorbed layer at the hydrophilic solid/liquid interface. Europhys. Lett. 67, 962-968 (2004).

21 Levine, J. R., Cohen, J. B., Chung, Y. W. \& Georgopoulos, P. Grazing-incidence smallangle X-ray scattering: new tool for studying thin film growth. J. Appl. Cryst. 22, 528 (1989).

22 Müller-Buschbaum, P., Vanhoorne, P., Scheumann, V. \& Stamm, M. Observation of nano-dewetting structures. Europhys. Lett. 40, 655-660 (1997).

23 Müller-Buschbaum, P., Hermsdorf, N., Roth, S. V., Wiedersich, J., Cunis, S. \& Gehrke, R. Comparative analysis of nanostructured diblock copolymer films. Spectrochim. Acta B 59, 1789-1797 (2004).

24 Wolff, M., Magerl, A. \& Zabel, H. Structure of polymer micelles close to the solid interface - a grazing incidence small angle neutron scattering study. Euro. Phys. J. E. $16,141-145$ (2005).

25 Förster, S., Timmann, A., Konrad, M., Schellbach, C., Meyer, A., Funari, S. S., Mulvaney, P. \& Knott, R. Scattering curves of ordered mesoscopic materials. J. Phys. Chem. B 109, 1347-1360 (2005).

26 Wolff, M., Magerl, A. \& Zabel, H. Structure of polymer micelles at the solid interface. Physica B 357, 84-87 (2005).

27 Steitz, R., Schemmel, S., Shi, H. W. \& Findenegg, G. H. Boundary layers of aqueous surfactant and block copolymer solutions against hydrophobic and hydrophilic solid surfaces. J. Phys. Condens. Matter 17, S665-S683 (2005).

28 Hamilton, W. A. Conformation, directed self-assembly and engineered modification: some recent near surface structure determinations by grazing incidence small angle X-ray and neutron scattering. Curr. Opin. Colloid. Interf. Sci. 9, 390-395 (2005).

29 Müller-Buschbaum, P., Maurer, E., Bauer, E. \& Cubitt, R. Surface versus confinement induced morphology transition in triblock copolymer films: A grazing incidence small angle neutron scattering investigation. Langmuir 22, 9295 (2006).

30 Kampmann, R., Haese-Seiller, M., Kudryashov, V., Nickel, B., Daniel, C., Fenzl, W., Schreyer, A., Sackmann, E. \& Rädler, J. Horizontal ToF-neutron reflectometer REFSANS at FRM-II Munich/Germany: first tests and status. Physica B 385-386, 1161-1163 (2006)

31 Walz, M., Magerl, A., Wolff, M. \& Zabel, H. Structure and texture of micelles under shear. Superlattice Microst. 41, 190-195 (2007).

32 Kentzinger, E., Frielinghaus, H., Rücker, U., loffe, A., Richter, D. \& Brückel, T. Probing lateral magnetic nanostructures by polarized GISANS. Physica B 397, 43-46 (2007).

33 Müller-Buschbaum, P., Schulz, L., Metwalli, E., Moulin, J. F. \& Cubitt, R. Lateral structures of buried interfaces in ABA-type triblock copolymer films. Langmuir 24, 7639-7644 (2008).

34 Cousin, F., Jestin, J., Chaboussant, G., Gautrot, S., Menelle, A. \& Ott, F. Probing simultaneously the volume and surface structure of nanospheres adsorbed at a solidliquid interface by GISANS. Euro. Phys. J. E. ST. 167, 177-183 (2009).

35 Müller-Buschbaum, P., Metwalli, E., Moulin, J. F., Kudryashov, V., Haese-Seiller, M. \& Kampmann, R. Time of flight grazing incidence small angle neutron scattering. Euro. Phys. J. E. ST. 167, 107-112 (2009).

36 Müller-Buschbaum, P., Schulz, L., Metwalli, E., Moulin, J. F. \& Cubitt, R. Interfaceinduced morphology transition in triblock copolymer films swollen with low-molecularweight homopolymer. Langmuir 25, 4235-4242 (2009).

37 Metwalli, E., Moulin, J. F., Gebhardt, R., Cubitt, R., Tolkach, A., Kulozik, U. \& MüllerBuschbaum, P. Hydration behavior of casein micelles in thin film geometry: a GISANS study. Langmuir 25, 4124-4134 (2009).

38 Avdeev, M. V., Bodnarchuk, V. I., Lauter-Pasyuk, V. V., Lauter, H., Aksenov, V. L., Yaradaikin, S. P., Ulyanov, V. A., Trounov, V. A. \& Kalinin, S. I. Project of the new multifunctional reflectometer GRAINS with horizontal sample plane at the IBR 2M pulsed reactor in Dubna. J. Phys. Conf. Ser. 251, 012060 (2010).

39 Kaune, G., Haese-Seiller, M., Kampmann, R., Moulin, J. F., Zhong, Q. \& MüllerBuschbaum, P. TOF-GISANS investigation of polymer infiltration in mesoporous TiO2 films for photovoltaic applications. J. Polym. Sci. Part B 48, 1628-1635 (2010).

40 Mitamura, K., Yamada, N. L., Sagehashi, H., Seto, H., Torikai, N., Sugita, T., Furusaka, M. \& Takahara, A. Advanced neutron reflectometer for investigation on dynamic/static structures of soft-interfaces in J-PARC. J. Phys. Conf. Ser. 272, 012017 (2011).

41 Busch, P., Rauscher, M., Moulin, J. F. \& Müller-Buschbaum, P. Debye-Scherrer rings from block copolymer films with powder-like order. J. Appl. Cryst. 44, 370-379 (2011).

42 Xia, X., Metwalli, E., Ruderer, M. A., Körstgens, V., Busch, P., Böni, P. \& MüllerBuschbaum, P. Nanostructured diblock copolymer films with embedded magnetic nanoparticles. J. Phys. Condens. Matter 23, 254203 (2011).

43 Chaboussant, G., Desert, S., Lavie, P. \& Brulet, A. PA2O: a new SANS and GISANS project for soft matter materials and magnetism. J. Phys. Conf. Ser. 340, 012002 (2012). 
44 Ruderer, M. A., Meier, R., Porcar, L., Cubitt, R. \& Müller-Buschbaum, P. Phase separation and molecular intermixing in polymer-fullerene bulk heterojunction thin films. J. Phys. Chem. Lett. 3, 683-688 (2012).

45 Korolkov, D., Busch, P., Willner, L., Kentzinger, E., Rucker, U., Paul, A., Frielinghaus, H. \& Bruckel, T. Analysis of randomly oriented structures by grazing-incidence smallangle neutron scattering. J. Appl. Cryst. 45, 245-254 (2012).

46 Yoneda, Y. Anomalous surface reflection of X-rays. Phys. Rev. 131, 2010 (1963).

47 Sinha, S. K., Sirota, E. B., Garoff, S. \& Stanley, H. B. X-ray and neutron scattering from rough surfaces. Phys. Rev. B 38, 2297 (1988).

48 Holý, V., Kubuena, J., Ohlídal, I., Lischka, K. \& Plotz, W. X-ray reflection from rough layered systems. Phys. Rev. B 47, 15896 (1993).

49 Holý, V. \& Baumbach, T. Non specular X-ray reflection from rough multilayers. Phys. Rev. B 49, 10668 (1994).
50 Baumbach, G. T., Holý, V., Pietsch, U. \& Gailhanou, M. The influence of specular interface reflection on grazing-incidence x-ray diffraction and diffuse-scattering from superlattices. Physica B 198, 249 (1994).

51 Kaune, G., Ruderer, M. A., Metwalli, E., Wang, W., Couet, S., Schlage, K., Röhlsberger, R., Roth, S. V. \& Müller-Buschbaum, P. In-situ GISAXS study of gold film growth on conducting polymer films. ACS Appl. Mater. Interfaces 1, 353 (2009).

52 James, R. W. The Optical Principles of the Diffraction of X-Rays (OxBow Press, Woodbridge Connecticut, 1962).

53 Wunnicke, O., Müller-Buschbaum, P., Wolkenhauer, M., Lorenz-Haas, C., Cubitt, R., Leiner, V. \& Stamm, M. Stabilization of thin polymeric bilayer films on top of semiconductor surfaces. Langmuir 19, 8511 (2003)

54 Müller-Buschbaum, P., Wolkenhauer, M., Wunnicke, O., Stamm, M., Cubitt, R. \& Petry, W. Structure formation in two-dimensionally confined diblock copolymer films. Langmuir 17, 5567 (2001).

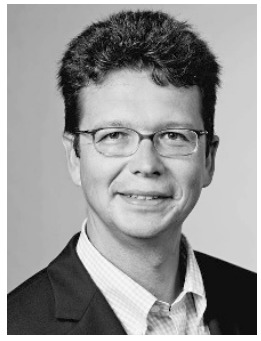

Peter Müller-Buschbaum is full university professor and heading the chair of Functional Materials in replacement of Professor Petry at the Physics department of Technische Universität München. He received his Doctorate Degree in Physics at Universität Kiel in 1996, followed by a postdoctoral work at Max-Planck-Institute for Polymer Research in Mainz. In 1999, he moved to the Physics department of Technische Universität München and joined the chair of Professor Winfried Petry on a group leader position. In 2002, he finished his habilitation and became assistant professor. Since 2006, he is full university professor. He is member of TUM.Energy, heading TUM.solar and the 'Network for Renewable Energy' (NRG) of the Munich School of Engineering (MSE). He is the German representative for Polymer physics in the European Polymer Federation (EPF) and member of several scientific advisory councils and panels. 